\title{
Safety and Efficacy of Renin-Angiotensin-Aldosterone System Inhibitors in COVID-19 Population
}

\author{
Yasar Sattar ${ }^{1} \cdot$ Pradeeksha Mukuntharaj $^{2} \cdot$ Mohamed Zghouzi $^{3} \cdot$ Abdul-Rahman M. Suleiman $^{3} \cdot$ Hassan Attique $^{4}$. \\ Waqas Ullah ${ }^{5} \cdot$ Muhammad Khawar Sana $^{6} \cdot$ Nathan Zaher $^{3} \cdot$ Maham Mehmood $^{7}$. Rajkumar P. Doshi ${ }^{8}$.

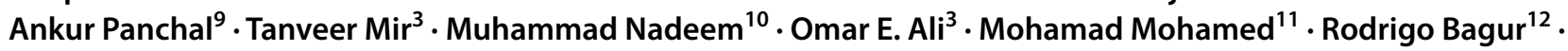 \\ Islam Y. Elgendy ${ }^{13} \cdot$ Mamas A. Mamas $^{11} \cdot$ M. Chadi Alraies ${ }^{3}$ (I)
}

Received: 20 March 2021 / Accepted: 19 May 2021 / Published online: 28 June 2021

(c) Italian Society of Hypertension 2021

\begin{abstract}
Introduction The safety of renin-angiotensin-aldosterone system inhibitors (RAASi) among COVID-19 patients has been controversial since the onset of the pandemic.

Methods Digital databases were queried to study the safety of RAASi in COVID-19. The primary outcome of interest was mortality. The secondary outcome was seropositivity improvement/viral clearance, clinical manifestation progression, and progression to intensive care units. A random-effect model was used to compute an unadjusted odds ratio (OR).

Results A total of 49 observational studies were included in the analysis consisting of 83,269 COVID-19 patients (RAASi $\mathrm{n}=34,691$; non-RAASi $\mathrm{n}=48,578$ ). The mean age of the sample was 64 , and $56 \%$ were males. We found that RAASi was associated with similar mortality outcomes as compared to non-RAASi groups (OR 1.07; 95\% CI 0.99-1.15; p >0.05). RAASi was associated with seropositivity improvement including negative RT-PCR or antibodies, (OR 0.96; 95\% CI $0.93-0.99 ; \mathrm{p}<0.05)$. There was no association between RAASi versus control with progression to ICU admission (OR 0.99; 95\% CI 0.79-1.23; $\mathrm{p}>0.05$ ) or higher odds of worsening of clinical manifestations (OR 1.04; 95\% CI 0.97-1.11; $\mathrm{p}>0.05$ ). Metaregression analysis did not change our outcomes for effect modifiers including age, sex, comorbidities, RAASi type, or study type on outcomes.

Conclusions COVID-19 is not a contraindication to hold or discontinue RAASi as they are not associated with higher mortality or worsening symptoms. Continuation of RAASi might be associated with favorable outcomes in COVID-19, including seropositivity/viral clearance.
\end{abstract}

Keywords Renin-angiotensin-aldosterone system inhibitors · RAASi · COVID-19 · Angiotensin-converting enzyme inhibitor · ACEi · Angiotensin receptor blocker · ARB

Yasar Sattar and Pradeeksha Mukuntharaj contributed equally to this manuscript.

M. Chadi Alraies

alraies@hotmail.com

1 Icahn School of Medicine at Mount, Sinai Elmhurst Hospital, Queens, NY, USA

2 Grant Medical College and Sir JJ Group of Hospitals, Mumbai, India

3 Detroit Medical Center, DMC Heart Hospital, 311 Mack Ave, Detroit, MI 48201, USA

4 University of Connecticut, Mansfield, CT, USA

5 Abington Jefferson Health, Abington, PA, USA

6 King Edward Medical University, Lahore, Punjab, Pakistan
7 Icahn School of Medicine at Mount Sinai BronxCare Health System, Bronx, NY, USA

8 University of Nevada School of Medicine, Reno, NV, USA

9 University of Pittsburgh Medical Center, Pittsburgh, PA, USA

10 University of Kentucky, Lexington, KY, USA

11 Keele Cardiovascular Research Group, Keele University, Stoke-on-Trent, UK

12 Western University, London, ON, Canada

13 Weill Cornell Medicine-Qatar, Doha, Qatar 


\section{Key Points}

RAASi of any type compared to placebo/non-RAASi does not affect mortality, severity, or ICU admissions in COVID 19 patients.

RAASi enhance viral clearance.

Age, sex, comorbidities, and RAASi type have no effect on the clinical outcomes in COVID 19 patients that are on RAASi.

\section{Introduction}

The coronavirus disease (COVID-19) pandemic caused by severe acute respiratory syndrome coronavirus 2 (SARS CoV2) has affected more than 80 million individuals worldwide, with 1.7 million deaths as of December 2020 [1]. The association of renin-angiotensin-aldosterone system inhibitors (RAASi) in the pathogenesis of COVID-19 has been well documented in the literature [2]. It has been widely accepted that COVID-19 uses angiotensin-converting enzyme two receptors (ACE2) to enter the cell [3-5]. COVID-19 can have fatal outcomes among patients with baseline comorbidities, including hypertension, heart failure, end-stage renal disease, chronic kidney disease, stroke, and chronic pulmonary diseases [2]. Patients with the comorbidities mentioned above are frequently on RAASi [2]. As a result, there have been concerns regarding the unintentional effect of RAASi's on adverse outcomes in COVID-19 [2,3]. However, several professional societies, including the International Society of Hypertension, the European Society of Hypertension, Joint American Heart Association, American College of Cardiology, and Heart Failure Society of United States, released a consensus statement supporting the continuing use of RAASi in patients [6-8].

To date, multiple meta-analyses have been conducted evaluating the association of ACEi and/or ARBs with COVID-19 outcomes [9-11]. While the results are consistent, many more extensive studies have since been conducted evaluating the effect of RAASi on many different outcomes in COVID-19 patients. Furthermore, findings from the recent BRACE CORONA trial also suggest that discontinuing RAASi does not affect the management of COVID-19 in hospitalized patients [12]. Because of this, we have designed this meta-analysis to provide an updated pooled quantitative estimate of the association between the use of RAASi and odds of COVID-19 mortality, seropositivity (SP)/viral clearance, the severity of clinical manifestations (SCM), and progression to intensive care unit (ICU).

\section{Methods}

The MEDLINE, PubMed, EMBASE, Cochrane Central Registry, Clinicaltrials.gov, and MedRxiv Preprint databases were queried using various combinations of medical subject headings (MeSH) to capture all studies on the safety and efficacy of RAASi in COVID-19 (Supplementary section 1). Databases were last assessed on November 14th, 2020. Two subsets of MeSH terms for COVID-19 and RAASi were combined using Boolean operators. All citations were downloaded to EndNote, and duplicates were removed. The titles and abstracts were searched for studies showing results of safety and efficacy of RAASi in COVID-19. There was no language or publication type filter applied for the search. Potentially relevant articles were read in full-text form, and desired outcomes of interest were extracted in an excel sheet. The primary outcome of interest was mortality. The secondary outcome was SP/ viral clearance, SCM, and progression to ICU. The standard definitions of variables of interest are given in supplementary section 2 . The inclusion criteria included any randomized trials or observational studies including patients with confirmed COVID-19 positive on oral/nasopharyngeal swab and compared the outcomes of those receiving any RAASi including ACE inhibitors or angiotensin II receptor blockers (ARBs) versus a control group with no RAASi use. Our study was conducted using MOOSE (Meta-analyses Of Observational Studies in Epidemiology) protocol and Preferred Reporting Items for Systematic Reviews and Meta-Analyses (PRISMA) [13, 14]. The detailed search strategy is showing in the PRISMA flow diagram (Fig. 1) (Supplementary section 1).

The statistical analysis was performed using a random effect model using meta size in STATA to calculate Odds ratios (OR) for binary outcomes (Supplementary section 3). The summary statistics analysis was performed by using a random effect model mean module in STATA (Supplementary section 4). The OR of binary outcomes was combined with the studies that had data available in precomputed effect size (OR, lower confidence interval [lci], and upper confidence interval [uci]). The OR, lci, uci were pooled using metan in STATA. The results were considered statistically significant if the probability value ( $p$ value) was less than 0.05 with a $95 \%$ confidence interval (CI) not crossing 1 . The test of overall effect was reported as Z-value with the association of $95 \%$ CI. Subgroup analysis was performed based on the type of RAASi or combination regimens. Higgin I-squared $\left(\mathrm{I}^{2}\right)$ statistical model was used to assess the variations in outcomes of interest. $\mathrm{I}^{2}$ values were divided into 0 to $25 \%, 25$ to $<75 \%$, and $>75 \%$ as mild, moderate, and severe heterogeneity, respectively. For outcomes with severe heterogeneity 
$\left(\mathrm{I}^{2}=>75 \%\right)$, we performed a jackknife sensitivity analysis, where the effect on $\mathrm{I}^{2}$ was noticed by removing one study at a time with a goal to bring to the lowest value [15] (Supplementary section 5). The publication bias was depicted graphically and numerically as forest plot and Egger's Regression Equation (ERE) (Supplementary section 6). Meta-regression was performed to see any potential effect modifiers using random effect models for study variance and Knapp-Hartung modification. [16-18] Univariate meta regression was performed on demographics (age, sex), comorbidities (hypertension, diabetes mellitus, chronic kidney disease, coronary artery disease, chronic obstructive pulmonary disease), the type of RAASi used, the type of study, and the following outcomes: severity, seropositivity, icu admission, and mortality (supplementary section 7). The quality of the included randomized trials was assessed using the Cochrane Quality Assessment tool, where individual studies were assessed for five different types of bias (selection, performance, detection, attrition, and reporting bias). The quality of observational studies was assessed by using Newcastle-Ottawa Scale (NOS) [19] (supplementary section 8). All statistical analysis was performed using STATA Version 16.1, College Station, TX: StataCorp LLC [20].

\section{Results}

\subsection{Search Results}

The initial search revealed 2078 numbers of articles. After excluding duplicates $(n=1589)$, the remaining articles were searched for the outcome of interest-based on title and abstract. After removing irrelevant items based on the title and abstract, 141 studies were deemed for full-text reading. A total of 49 numbers of articles were used in quantitative analysis [12, 21-69]. The reason for article inclusion and exclusion is shown in the PRISMA flow (Fig. 1).

\subsection{Study Characteristics}

A total of 49 studies with 83,269 of COVID-19 patients (RAASi $n=34,691$; non-RAASi $n=48,578$ ) were included in the study [12, 21-69]. The included study population had confirmed COVID-19 diagnosis by nasopharyngeal reverse transcriptase-polymerase chain reaction (RT-PCR). The mean age of the study population was 64 years, $56 \%$ were males, and the most common comorbidity or indication for RAASi was hypertension. The doses of RAASi were not presented in the majority of studies, but over 40 articles

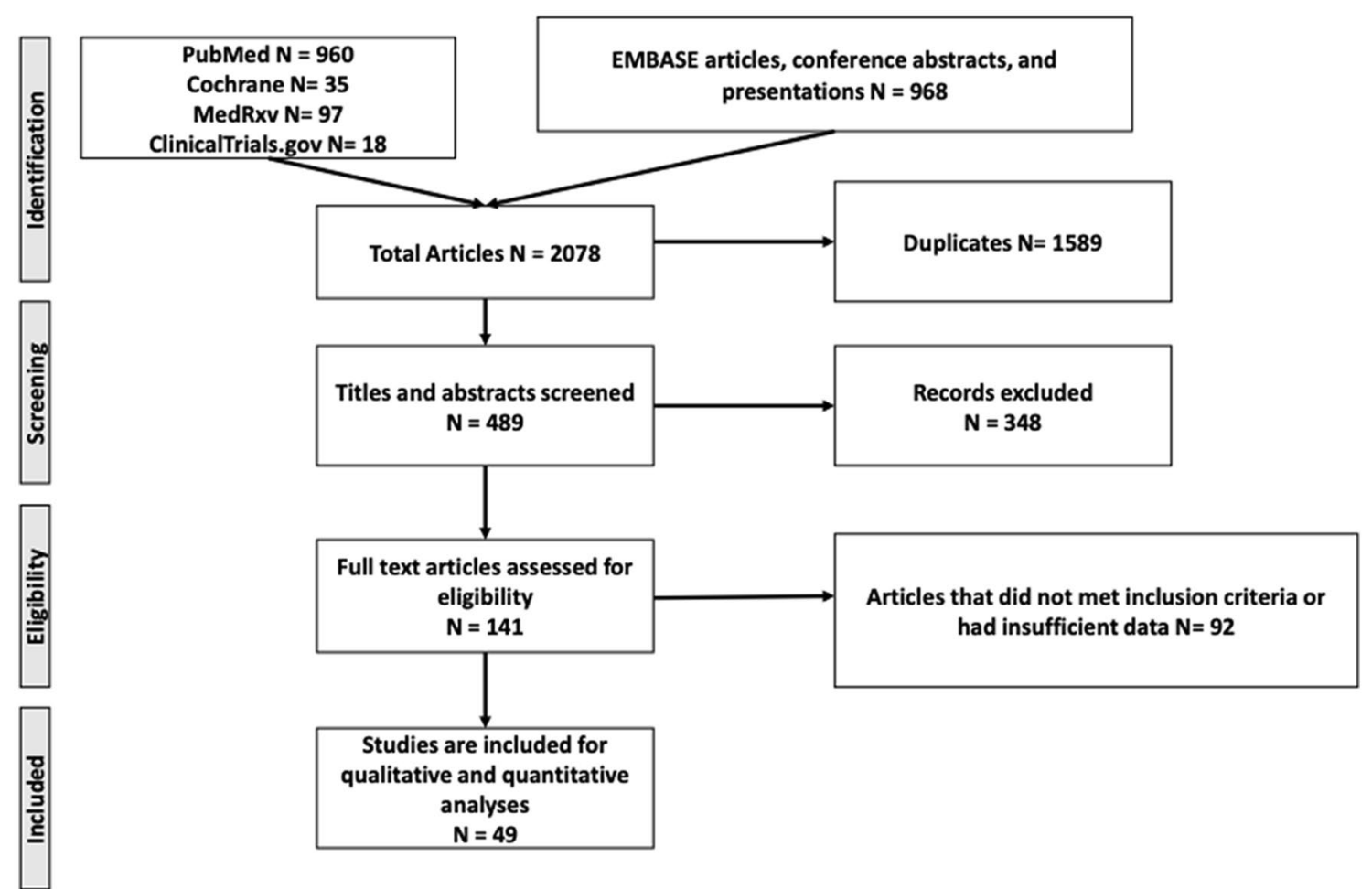

Fig. 1 PRISMA strategy for systematic review and meta-analysis 
studied combined data of ACEi and ARBs while the remaining 9 studies reported data for ACEi and ARBs separately. A completed baseline demographics and comorbidities of the included studies are shown in Table 1.

All included studies were observational, including 41 retrospective and 8 prospective in total. As randomization may not be achievable in observational designs, many studies' quality may be moderate at best. However, few studies used propensity matching for subjects to attempt minimizing selection bias. Reporting bias across most of the studies was reduced due to an adequate description of the study results. The risks of bias graph and summary are shown in Supplementary section 8 . Furthermore, The Newcastle-Ottawa Scale (NOS) was also utilized to evaluate the included publications' methodological quality. The NOS features eight criteria and yields scores ranging from 0 (high risk of bias) to 9 (low risk of bias). Studies with NOS scores of $>7$ were regarded as high quality (Supplementary section 8). Using NOS, 13 of the studies were considered moderate or low quality.

\subsection{Pooled Results}

On random effect analyses, RAASi use was not associated with an increased odds of mortality as compared to non RAASi groups in COVID-19 patients (OR 1.07; 95\% CI 0.99-1.15; $\mathrm{p}>0.05$ ) (32 studies, RAASi $\mathrm{n}=10,850$, nonRAASi $n=14,914 ; I^{2}=67.4 \%$ ) (Fig. 2). RAASi use was associated with seropositivity improvement, including negative RT-PCR or antibodies, (OR 0.96; 95\% CI $0.93-0.99 ; \mathrm{p}<0.05$ ) (14 studies, RAASi $\mathrm{n}=7859$, nonRAASi $\mathrm{n}=25,911 ; \mathrm{I}^{2}=88.0 \%$ ) (Fig. 2). RAASi use was not associated with worsening clinical manifestations (OR 1.04; 95\% CI 0.97-1.11; $\mathrm{p}>0.05$ ) (22 studies, RAASi $\mathrm{n}=12,622$, nonRAASi $\left.\mathrm{n}=26,450 ; \mathrm{I}^{2}=77.2 \%\right)$ or increased odds of ICU admission (OR 0.99; 95\% CI 0.79-1.23; p > 0.05) (6 studies, RAASi $n=2378$, nonRAASi $n=5188 ; I^{2}=81.0 \%$ ) (Fig. 3).

\subsection{Subgroup Analysis}

A subgroup analysis ( $\mathrm{SG}$ ) was performed to delineate the results with combination of ACEi and ARBs (RAASi $\mathrm{n}=17,801$, nonRAASi $\mathrm{n}=37,817$ ) or lone use of ACEi or ARBs (RAASi $\mathrm{n}=16,890$, nonRAASi $\mathrm{n}=10,761$ ) in COVID-19 patients. The SG results were synonymous to pooled outcomes except ICU admission (ARBs OR 1.56; 95\% CI 1.02-2.39; $\mathrm{p}<0.05$ ) (6 studies, ACEi or ARB $\mathrm{n}=144, \mathrm{ACEi}+\mathrm{ARB} \mathrm{n}=2234$ ), and seropositivity conversion (ACEi OR 0.96; 95\% CI 0.73-1.26; $p>0.05$ and ARBs OR 1.21; 95\% CI 0.96-1.53; p > 0.05) (14 studies, ACEi or $\mathrm{ARB} n=278$, ACEi $+\mathrm{ARB} n=7581$ ) as compared to combination of ARBs and ACEi (Supplementary Sect. 3).
Univariate regression for COVID patients taking RAASi showed no evidence of age, sex, hypertension, diabetes, chronic kidney disease, coronary artery disease, COPD, RAASi type, or study type having an effect on outcomes (i.e., mortality, seropositivity, ICU admission, and severity) (all P >0.05) (Table 2, supplemental file section 7).

A sensitivity analysis was performed for outcomes with high heterogeneity $\left(\mathrm{I}^{2}>75 \%\right)$. In terms of ICU admissions, high heterogeneity $\left(\mathrm{I}^{2}=81 \%\right)$ was likely secondary to Bean et al. After removal; the heterogeneity was $<75 \%$ $\left(\mathrm{I}^{2}=34.9 \%\right)$. The high heterogeneity $\left(\mathrm{I}^{2}=88 \%\right)$ with seropositivity was likely due to Khawaja et al. de Abajo et al. and Yan et al. After removal of both studies; heterogeneity was $<75 \%\left(\mathrm{I}^{2}=46.7 \%\right)$. In terms of severity, the heterogeneity was high $\left(\mathrm{I}^{2}=77.2 \%\right)$, likely due to studies by Bean et al. and Feng et al. After removal of both studies, heterogeneity was $<75 \%\left(\mathrm{I}^{2}=66.3 \%\right)$ (Supplementary section 5$)$.

The funnel plot's visual assessment was symmetrical, ruling out any visible publication bias (Supplementary section 6) The numerical assessment of publication bias was done using Egger's regression model that also failed to show any publication bias or small study effects $(E R E \approx>0.05)$. The limited scatter seen on the funnel plot can be secondary to variation in the cohort selection for individual study.

\section{Discussion}

In this updated meta-analysis of 49 articles with $>83 \mathrm{~K}$ COVID-19 patients, we found that the use of RAASi was not associated with increased short-term mortality. RAASi use was associated with improved seropositivity, a form of indirect evidence of viral clearance. RAASi use was also not associated with increased risk of admission to ICU or worsening of clinical manifestations. Collectively, these findings support the recommendations by professional societies for continuing RAASi irrespective of COVID19 infection [70].

The use of RAASi in COVID-19 has been controversial since the start of the pandemic, with some studies hypothesizing that the use of RAASi may increase the expression of the ACE2 receptor, the principal receptor for viral entry into the host cell [71]. Although pre-existing cardiovascular disease increased the severity and mortality associated with COVID-19 infection, we have found that the use of ACEi/ARBs is likely not a contributing factor. Our analysis summarized the available studies with the effect of ACEi or ARBs or ACEi + ARBs on COVID-19 outcomes to analyze protective or detrimental effects against severe COVID-19 infection.

In our study, RAASi use was not associated with an increased risk of mortality. BRACE CORONA is the only 


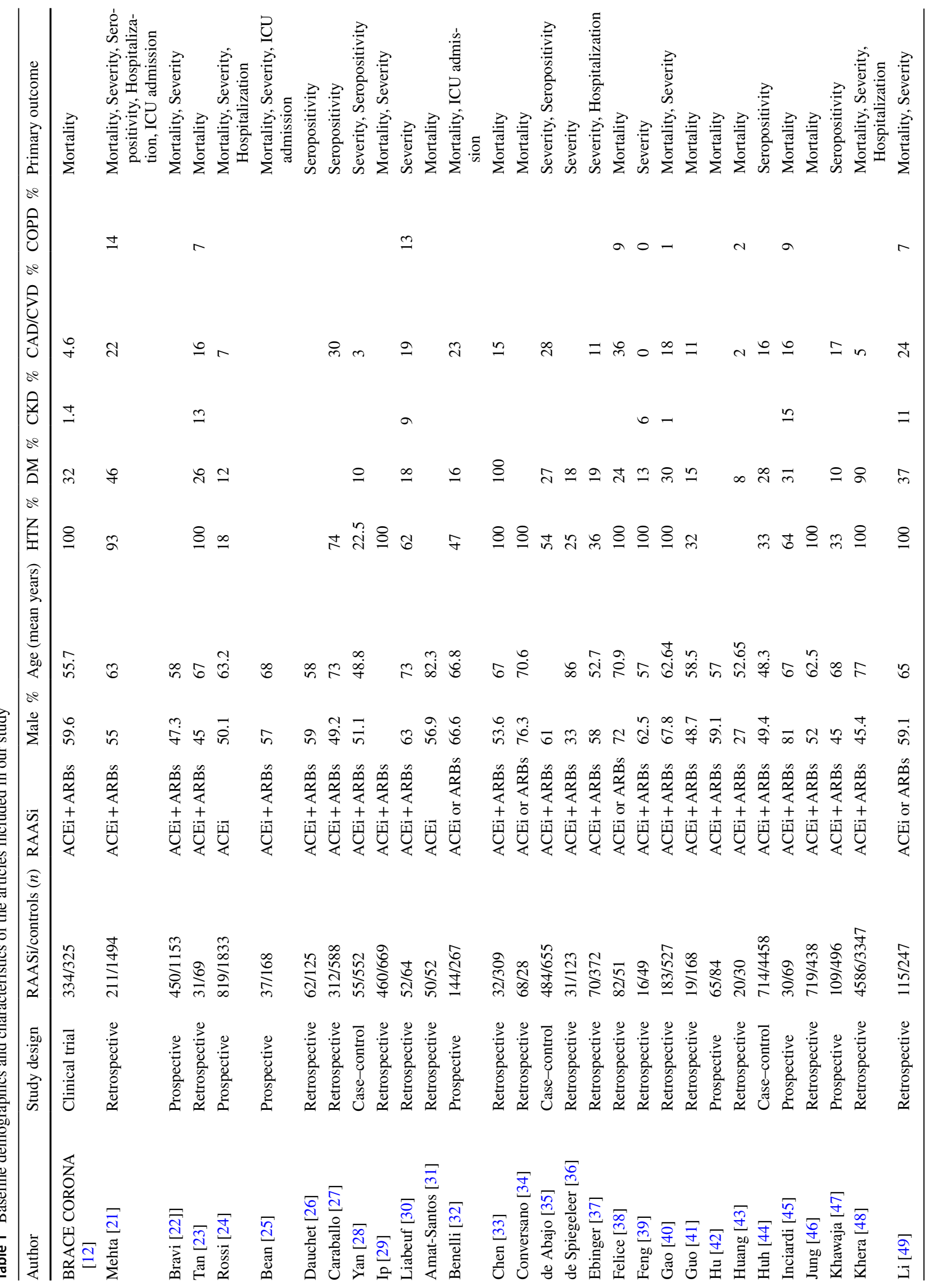


410

Y. Sattar et al.

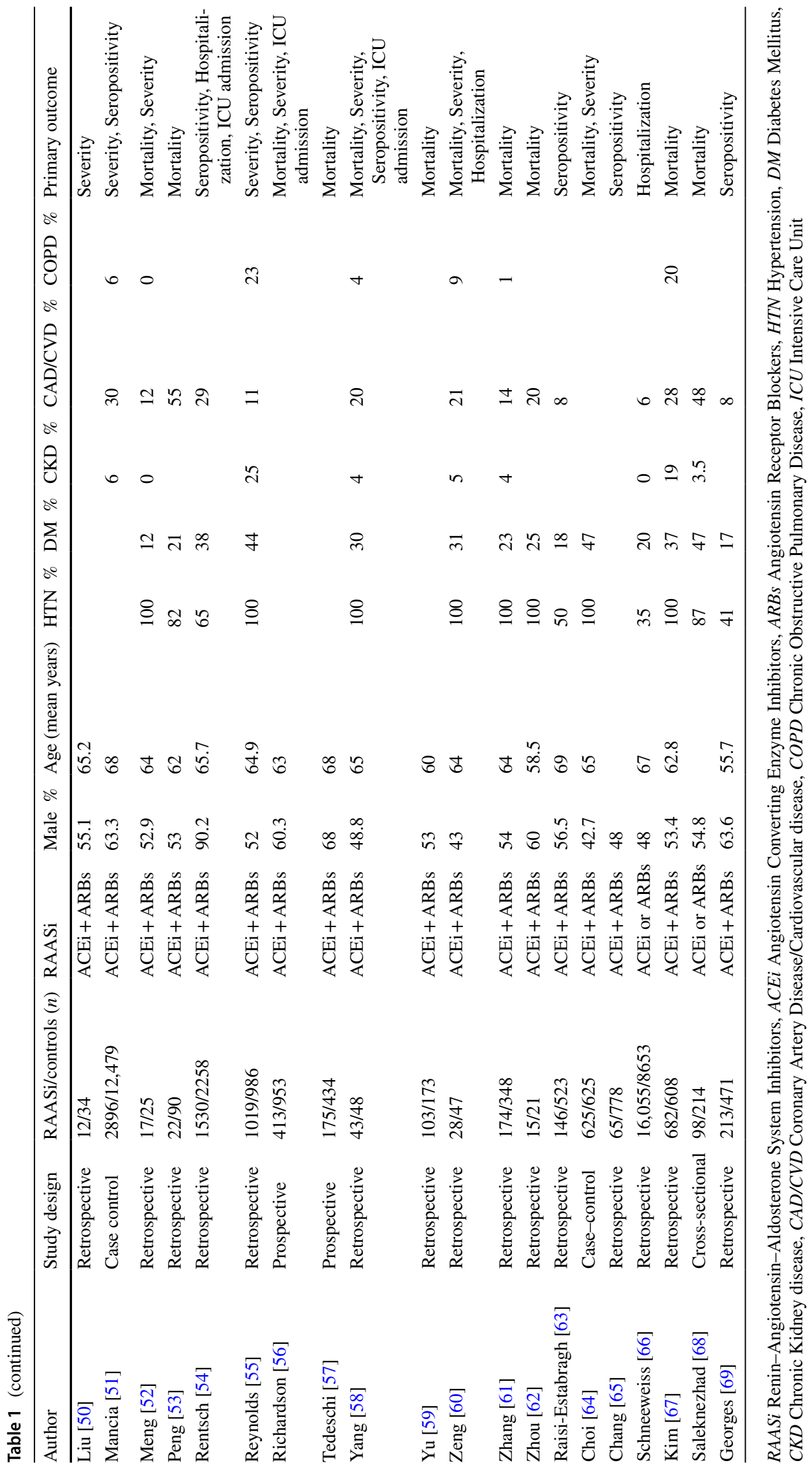

$\triangle$ Adis 

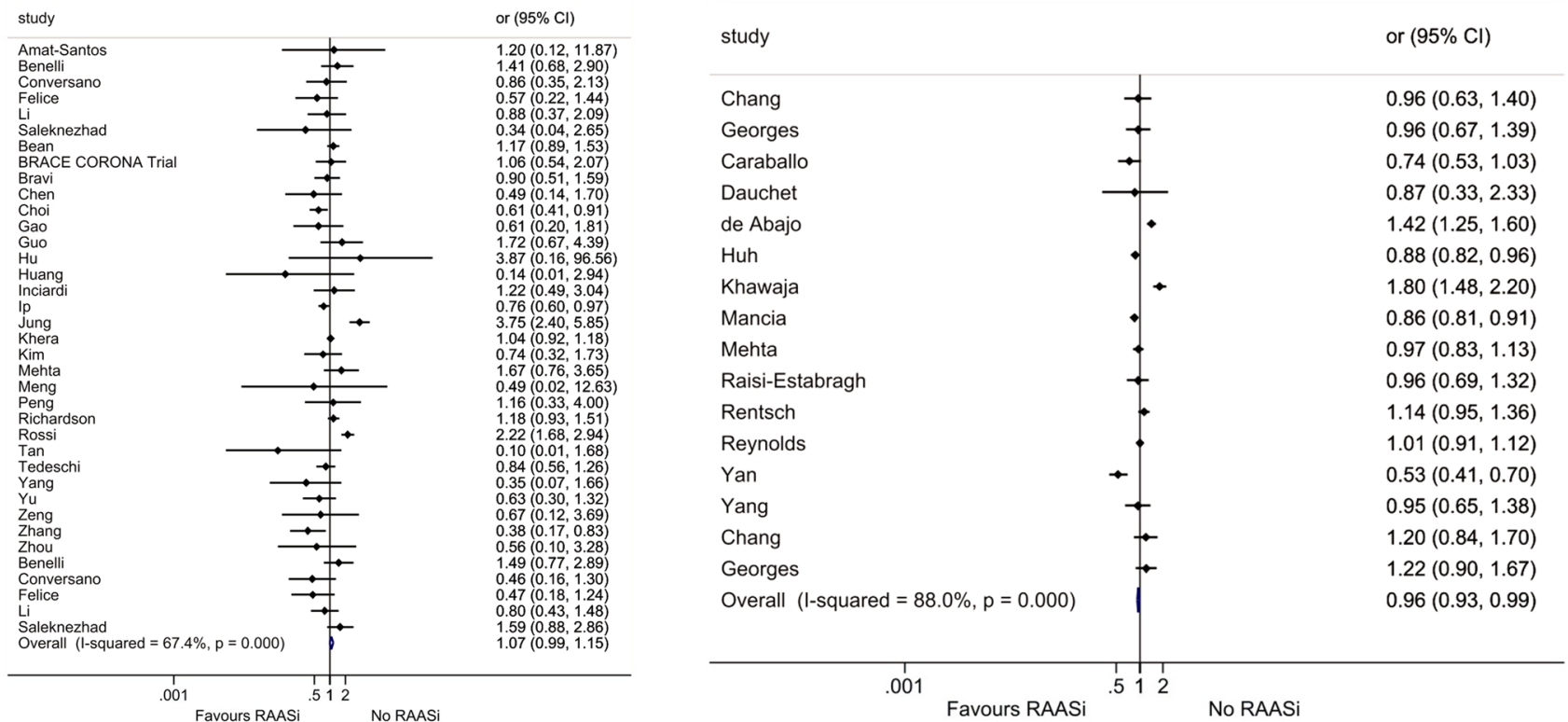

Fig. 2 Forest plots showing pooled mortality outcomes (left) and seropositivity rates (right) among COVID-19 with RAASi
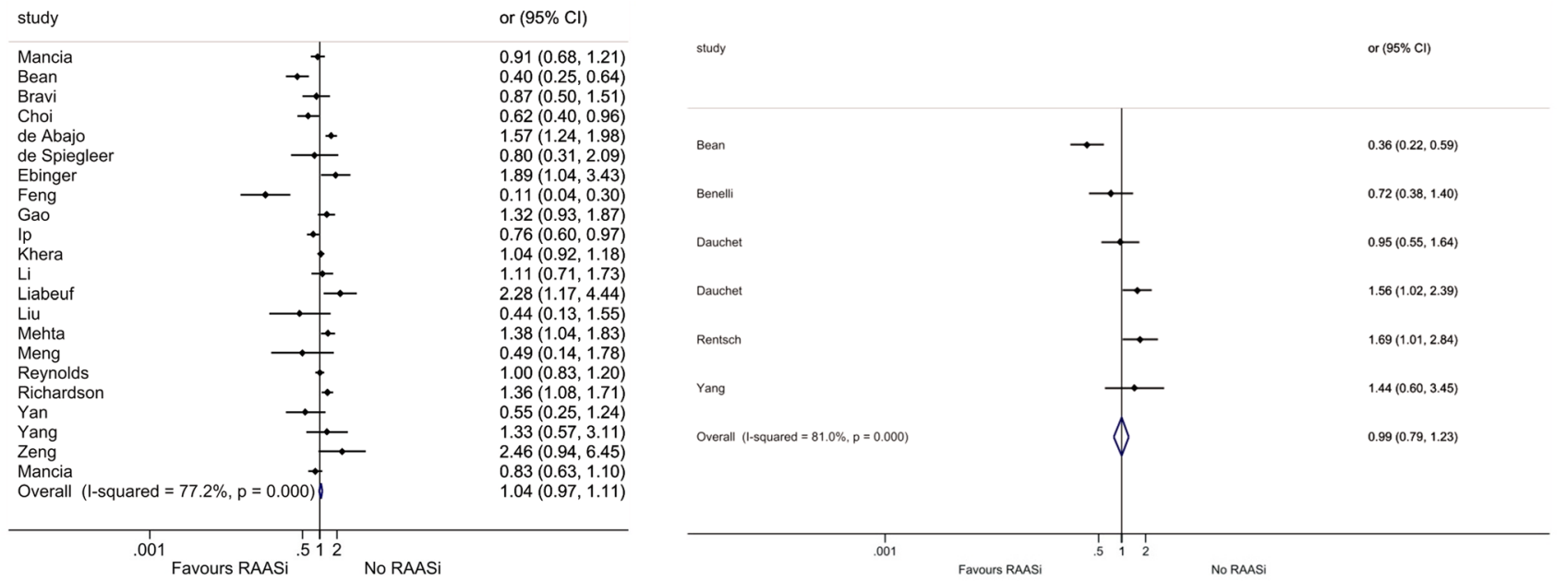

Fig. 3 Forest plot showing pooled severity outcomes (left) and ICU admission rates (right) among COVID -19 patients on RAASi and No RAASi

randomized control trial to date. Inclusion criteria included any 18 year or older patient with COVID 19 that requires hospitalization who are chronically on ACEi/ARBs. Patients were randomized to either continue ACEi/ARBs treatment or suspend the medication. Patients were followed for 30 days. The primary endpoint was the number of days alive and out of the hospital; the secondary endpoint was all causes of death. The study showed suspending ACEi/ARBs showed no benefits, there was no difference in days alive or out of the hospital and all causes of death at 30 days were the same.
With respect to ICU admissions, there was a significant increase in ICU admission odds with ARBs but not in combination with ACEi. Furthermore, ACEi alone did not affect ICU admission. There were relatively few studies that looked at COVID-19 ICU admission in patients taking RAASi. In a study by Bean et al., 14\% (5/37) of patients with exposure to an ACEi died or required critical care support compared to $29 \%$ (48/168) for patients without such exposure [25]. Bean et al. study findings suggested that ACEi did not increase the severity of COVID-19 as hypothesized but may instead 
Table 2 Meta-regression of potential effect modifiers for all study outcomes $(\mathrm{P}>0.05$ shows that our study had no effect modifiers in our studied outcomes)

\begin{tabular}{|c|c|c|c|c|c|c|c|c|}
\hline \multirow{2}{*}{$\begin{array}{l}\text { Meta-regres- } \\
\text { sion variables }\end{array}$} & \multicolumn{2}{|l|}{ Mortality } & \multicolumn{2}{|l|}{ Severity } & \multicolumn{2}{|c|}{ ICU admission } & \multicolumn{2}{|c|}{ Seropositivity } \\
\hline & Coefficient & $\mathrm{p}$ value & Coefficient & $\mathrm{p}$ value & Coefficient & $\mathrm{p}$ value & Coefficient & $\mathrm{p}$ value \\
\hline Age & 0.00 & 0.915 & 0.00 & 0.569 & -0.06 & 0.193 & 0.01 & 0.259 \\
\hline Male & 0.00 & 0.846 & 0.00 & 0.498 & 0.02 & 0.355 & 0.00 & 0.731 \\
\hline HTN & - & - & 0.00 & 0.538 & 0.02 & 0.363 & 0.00 & 0.857 \\
\hline $\mathrm{DM}$ & 0.00 & 0.727 & 0.00 & 0.486 & 0.04 & 0.055 & 0.00 & 0.830 \\
\hline CKD & 0.00 & 0.830 & 0.01 & 0.764 & - & - & - & - \\
\hline CAD & -0.01 & 0.549 & - & - & 0.08 & 0.488 & 0.01 & 0.487 \\
\hline COPD & 0.03 & 0.165 & 0.03 & 0.184 & - & - & - & - \\
\hline RAASi Type & 0.05 & 0.648 & 0.03 & 0.892 & -0.11 & 0.727 & -0.02 & 0.894 \\
\hline Study Type & -0.13 & 0.143 & 0.01 & 0.954 & - & - & -0.24 & 0.050 \\
\hline
\end{tabular}

$*=\mathrm{p}<0.05$

$H T N$ Hypertension, DM Diabetes Mellitus, CKD Chronic Kidney Disease, CAD Coronary artery disease, $C O P D$ Chronic obstructive pulmonary disease, RAASi Renin-Angiotensin-Aldosterone system inhibitors reduce the severity. Our meta-analysis reaffirms Bean et al.'s findings. Notably, Bean et al. could not comment on ARBs users owing to the small number of patients in this subgroup. Furthermore, their study had a short follow-up period, which might have led to missing late outcomes. In Dauchet et al., a statistically significant increase in the Standardised Prevalence Ratio (SPR) of ARBs (1.56 [1.02-2.39]) was observed in intensive care unit patients only while SPRs of $\mathrm{ACEi}+\mathrm{ARBs}$ did not differ as the severity of the COVID19 patients increased [26]. The results from these studies further reinforce the need to continue ACEi and ARBs in combination or ACEi's alone in COVID-19 patients. Additionally, it is important to note that when Dauchet et al. controlled for BMI, they found that ARB's were associated with an increase in SPR only in obese patients [26]. As a result, they hypothesize that the increase in SPR is likely due to a higher frequency of deleterious comorbidities on COVID19 outcomes in Obese patients [26]. Combined with the small sample size of ARB only use on ICU admission in our sample, it is likely that these findings are clinically insignificant.

In our study, ACEi and ARBs use did not increase the chances of testing positive for COVID-19 but instead seem to show a slight beneficial effect. Interestingly, the beneficial effect of RAASi on seropositivity was only present in patients who took ACEi and ARB's in combination. Many studies on seropositivity showed similar results [27, 59, 66]. But, a study by Caraballo et al. revealed that compared to patients on ACEi/ARBs, those not on these therapies were $50 \%$ more likely to be COVID-19 positive [27]. However, the study only included patients that tested positive for COVID-19, whereas those who did not wish to be tested or were asymptomatic were missed, which might have led to such a significant finding in ACEi/ARBs group. Yan et al. found that individuals with hypertension taking CCBs had significantly increased risk [odds ratio $(\mathrm{OR})=1.67(95 \% \mathrm{CI}$ 1.2-2.9)] of manifesting symptoms of COVID-19 whereas those taking ARBs had significantly lower disease risk (OR 0.24 ; 95\% CI 0.17-0.34 and OR 0.32; 95\% CI 0.19-0.57 respectively) [28]. Although this was a large populationbased study, the retrospective nature may have led to underreporting of symptoms and drug history. Nevertheless, the results are significant and further confirm the neutral effect of ACEi and ARBs in patients with a COVID-19 positive test. Of note, a potential explanation for the beneficial effect of RAASi use on seropositivity in our sample may be due to a decrease in the activation of the RAAS leading to a decreased inflammatory response and lower odds of cytokine storm which has been implicated in the pathogenesis of COVID-19 related lung injury [61].

Several studies were performed to evaluate the association between ACEi/ARBs use and COVID-19. A retrospective study by Ip et al. found that there were lower mortality rates in hypertensive patients prescribed ACEi $(27 \%$, $\mathrm{p}<0.01)$ or ARBs $(33 \%, \mathrm{p}<0.05)$ compared to other antihypertensive agents (39\%) [29]. RAASi appeared protective compared to other anti-hypertensive agents $(\mathrm{p}=0.001)$. This study carried the usual limitations of a retrospective study but was a multicenter study with a significant result on the outcome's severity. In another study by Liabeuf et al., the risk of severe COVID-19 was higher in patients treated with a RAASi (OR 1.97, 95\% CI 1.03-3.78) than in patients not treated with a RAASi [30]. Known confounders were eliminated by adjusting for age and hypertension, but there might be a risk for unknown confounders. However, this study included only hospitalized patients; therefore, their definition of non-severe COVID-19 cannot be applied to the general population. Our meta-analysis results found no evidence of an association between severity of COVID-19 infection and ACEi/ARBs use (OR 1.04; 95\% CI 0.97-1.11; $\mathrm{p}>0.05)$. This is in congruence with previous studies and the society's recommendations of continuing ACEi and ARBs irrespective of COVID-19 positivity [72]. 
It is important to note, several of our analyses had to be refined due to high heterogeneity. For example, we removed Bean et al. from the analysis of our ICU admissions outcome to reduce heterogeneity. Bean et al. was likely driving the high heterogeneity due to a large number of patients with severe outcomes, such as organ support [25]. We removed Khawaja et al., Yan et al., and de Abajo et al. to reduce heterogeneity for seropositivity. Khawaja's study likely underascertained COVID-19 cases as severe and mild [47]. Yan et al. also included patients taking antihypertensives other than ACEi/ARB [28]. Additionally, de Abajo et al. used different databases for cases and controls, which could have led to information bias and incorrect results [35]. Finally, high heterogeneity in our severity outcome was due to Feng et al. and Bean et al. This is likely because there was an increased number of missing data with Feng et al. and inclusion of patients aged $>80$ with Bean et al. (supplementary file section 5) [25, 39].

The key strength of our study lies in the large sample size and comprehensive search. Furthermore, our analysis of comorbidities and effect sizes for both individual treatment and co-administration of ACEi and ARBs could be weighed, which aids in comparing the outcomes in each subgroup. As randomized clinical trials continue to progress, the association between COVID-19 and RAASi will become clearer. [73-78].

\subsection{Limitations}

The results of our meta-analysis should be interpreted in the context of some limitations. Many studies included in the meta-analysis were observational studies and also included pre-printed versions. Most of the studies are subjective to selection bias secondary to a failure of randomization. There was also a risk of attribution bias and a loss of clinical follow-up. Furthermore, due to a lack of blinding, we could not assess for unknown confounders. Several studies did not match for confounders, which may affect the power of the study. These confounders could change the strength of association between COVID-19 infection and RAASi. Nevertheless, the use of ACEi and ARBs conferred an overall protective benefit for COVID-19 patients. Additionally, high heterogeneity required us to omit several studies from our analysis. Finally, meta regression analysis based on obesity/BMI was not performed due to a limitation in the data leading to linear regression convergence being unable to be achieved. Similarly, the effect of hypertension on mortality, coronary artery disease on severity, both chronic kidney disease and COPD on ICU admission and seropositivity, and study type on ICU admission could not be performed due to a limitation in the data leading to linear regression convergence being unable to be achieved.

\section{Conclusion}

RAASi use is not associated with increased short-term mortality, severity, or ICU admissions. Thus, our results reinforce the current recommendations by the ESH, HFSA/ACC/ AHA against discontinuing ACEi/ARBs in the patients who were previously on these therapies.

Supplementary Information The online version contains supplementary material available at https://doi.org/10.1007/s40292-021-00462-w.

\section{Declarations}

Funding None.

Conflict of interest Not applicable.

Ethics approval Not applicable.

Consent to participate Not applicable.

Consent to publish Not applicable.

Availability of data and material Not applicable.

Code availability Not applicable.

Author contributions Not applicable.

\section{References}

1. COVID-19 Map-Johns Hopkins Coronavirus Resource Center [Internet]. Johns Hopkins Coronavirus Resource Center.: https:// coronavirus.jhu.edu/map.html.

2. Sattar Y, Ullah W, Rauf H, Virk HUH, Yadav S, Chowdhury M, Connerney M, Mamtani S, Pahuja M, Patel RD, Mir T, Almas T, Moussa Pacha H, Chadi Alraies M. COVID-19 cardiovascular epidemiology, cellular pathogenesis, clinical manifestations and management. Int J Cardiol Heart Vasc. 2020;29:100589. https:// doi.org/10.1016/j.ijcha.2020.100589. PMID: 32724831; PMCID: PMC7359794.

3. Oudit G, Kassiri Z, Jiang C, Liu P, Poutanen S, Penninger J, Butany J. SARS-coronavirus modulation of myocardial ACE2 expression and inflammation in patients with SARS. Eur J Clin Invest. 2009;39:618-25.

4. Negreira-Caamaño M, Piqueras-Flores J, Martínez-DelRio J, Nieto-Sandoval-Martin-DeLaSierra P, Aguila-Gordo D, MateoGomez C, Salas-Bravo D, Rodriguez-Martinez M, NegreiraCaamaño M. Impact of treatment with renin-angiotensin system inhibitors on clinical outcomes in hypertensive patients hospitalized with COVID-19. High Blood Press Cardiovasc Prev. 2020;27(6):561-568.

5. Vaduganathan M, Vardeny O, Michel T, McMurray JJV, Pfeffer MA, Solomon SD. Renin-angiotensin-aldosterone system inhibitors in patients with Covid-19. N Engl J Med. 2020;382(17):1653-9.

6. Position Statement of the ESC Council on Hypertension on ACEInhibitors and Angiotensin Receptor Blockers. https://www.escar dio.org/Councils/Council-on-Hypertension-(CHT)/News/posit 
ion-statement-of-the-esc-council-on-hypertension-on-ace-inhib itors-and-ang.

7. HFSA/ACC/AHA Statement Addresses Concerns Re: Using RAAS Antagonists In COVID-19-American College Of Cardiology. https://www.acc.org/latest-in-cardiology/articles/2020/ 03/17/08/59/hfsa-acc-aha-statement-addresses-concerns-re-usingraas-antagonists-in-covid-19.

8. Iaccarino G, Borghi C, Cicero AFG, Ferri C, Minuz P, Muiesan ML, Mulatero P, Mulè G, Pucci G, Salvetti M, Savoia C, Sechi LA, Volpe M, Grassi G. Renin-angiotensin system inhibition in cardiovascular patients at the time of COVID19: much ado for nothing? A statement of activity from the directors of the board and the scientific directors of the italian society of hypertension. High Blood Press Cardiovasc Prev. 2020;27(2):105-8.

9. Pranata R, Permana H, Huang I, Lim MA, Soetedjo NNM, Supriyadi R, Soeroto AY, Alkatiri AA, Firman D, Lukito AA. The use of renin angiotensin system inhibitor on mortality in patients with coronavirus disease 2019 (COVID-19): a systematic review and meta-analysis. Diabetes Metab Syndrome Clin Res Rev. 2020;14:983-90.

10. Grover A, Oberoi M. A systematic review and meta-analysis to evaluate the clinical outcomes in COVID-19 patients on angiotensin converting enzyme inhibitors or angiotensin receptor blockers. medRxiv 2020.

11. Baral R, White M, Vassiliou VS. Effect of renin-angiotensinaldosterone system inhibitors in patients with COVID-19: a systematic review and meta-analysis of 28,872 patients. Curr Atheroscler Rep. 2020;22:1-9.

12. Lopes RD, et al. Effect of discontinuing vs continuing angiotensin-converting enzyme inhibitors and angiotensin ii receptor blockers on days alive and out of the hospital in patients admitted with COVID-19: a randomized clinical trial. JAMA. 2021;325(3):254-64.

13. MOOSE (Meta-analyses Of Observational Studies in Epidemiology) Checklist. https://www.elsevier.com/__data/promis_misc/ ISSM MOOSE Checklist.pdf.

14. Moher D, Liberati A, Tetzlaff J, Altman DG, Group P. Preferred reporting items for systematic reviews and meta-analyses: the PRISMA statement. PLoS Med. 2009;6:

15. Miller RG. The jackknife—a review. Biometrika. 1974;61:1-15.

16. Hartung J. An alternative method for meta-analysis. Biometr J. 1999;41:901-16.

17. Hartung J, Knapp G. On tests of the overall treatment effect in meta-analysis with normally distributed responses. Stat Med. 2001;20:1771-82.

18. Hartung J, Knapp G. A refined method for the meta-analysis of controlled clinical trials with binary outcome. Stat Med. 2001;20:3875-89.

19. Wells G. The Newcastle-Ottawa Scale (NOS) for assessing the quality of nonrandomised studies in meta-analysis. http://www. ohrica/programs/clinical_epidemiologyoxford.htm 2004.

20. StataCorp L. Stata Statistical Software. Release 16.[software]. College Station, TX: Stata Press. https://www.stata.com/. Accessed Sep 2019.

21. Mehta N, Kalra A, Nowacki AS, Anjewierden S, Han Z, Bhat P, Carmona-Rubio AE, Jacob M, Procop GW, Harrington S, Milinovich A, Svensson LG, Jehi L, Young JB, Chung MK. Association of Use of Angiotensin-Converting Enzyme Inhibitors and Angiotensin II Receptor Blockers With Testing Positive for Coronavirus Disease 2019 (COVID-19). JAMA Cardiol. 2020 Sep 1;5(9):1020-1026. https://doi.org/10.1001/jamacardio.2020. 1855. PMID: 32936273; PMCID: PMC7201375.

22. Bravi F, Flacco ME, Carradori T, Volta CA, Cosenza G, De Togni A, Martellucci CA, Parruti G, Mantovani L, Manzoli L. Predictors of severe or lethal COVID-19, including angiotensin converting enzyme inhibitors and angiotensin II receptor blockers, in a sample of infected Italian citizens. medRxiv. 2020.

23. Tan N-D, Qiu Y, Xing X-B, Ghosh S, Chen M-H, Mao R. Associations between angiotensin-converting enzyme inhibitors and Angiotensin II Receptor blocker use, gastrointestinal symptoms, and mortality among patients with COVID-19. Gastroenterology. 2020;159(1170-1172):

24. Rossi PG, Marino M, Formisano D, Venturelli F, Vicentini M, Grilli R, Group REC-W. Characteristics and outcomes of a cohort of SARS-CoV-2 patients in the Province of Reggio Emilia, Italy. medRxiv. 2020.

25. Bean D, Kraljevic Z, Searle T, Bendayan R, Pickles A, Folarin A, Roguski L, Noor K, Shek A, Zakeri R. Treatment with ACE-inhibitors is associated with less severe disease with SARS-Covid-19 infection in a multi-site UK acute Hospital Trust. medRxiv. 2020.

26. Dauchet L, Lambert M, Gauthier V, Poissy J, Faure K, Facon A, Yelnik C, Panaget S, Plagnieux T, Verfaillie F. ACE inhibitors, AT1 receptor blockers and COVID-19: clinical epidemiology evidences for a continuation of treatments. The ACER-COVID study. medRxiv. 2020.

27. Caraballo C, McCullough M, Fuery M, Chouairi F, Keating C, Ravindra N, Miller E, Malinis M, Kashyap N, Hsiao A. COVID19 infections and outcomes in a live registry of heart failure patients across an integrated health care system. medRxiv. 2020.

28. Yan H, Valdes AM, Vijay A, Wang S, Liang L, Yang S, Wang $\mathrm{H}$, Tan X, Du J, Jin S. Role of drugs affecting the renin-angiotensin-aldosterone system on susceptibility and severity of COVID-19: A large case-control study from Zheijang Province, China. medRxiv. 2020.

29. Ip A, Parikh K, Parrillo JE, Mathura S, Hansen E, Sawczuk IS, Goldberg SL. Hypertension and renin-angiotensin-aldosterone system inhibitors in patients with Covid-19. medRxiv. 2020.

30. Liabeuf S, Moragny J, Bennis Y, Batteux B, Brochot E, Schmit JL, Lanoix J-P, Andrejak C, Ganry O, Slama M. Association between renin-angiotensin system inhibitors and COVID-19 complications. Eur Heart J Cardiovasc Pharmacother. 2020.

31. Amat-Santos IJ, Santos-Martinez S, López-Otero D, NombelaFranco L, Gutiérrez-Ibanes E, Del Valle R, Muñoz-García E, Jiménez-Diaz VA, Regueiro A, González-Ferreiro R. Ramipril in High Risk Patients with COVID-19. J Am Coll Cardiol. 2020.

32. Benelli G, Buscarini E, Canetta C, La Piana G, Merli G, Scartabellati A, Vigano G, Sfogliarini R, Melilli G, Assandri R. SARS-COV-2 comorbidity network and outcome in hospitalized patients in Crema, Italy. medRxiv 2020.

33. Chen Y, Yang D, Cheng B, Chen J, Peng A, Yang C, Liu C, Xiong M, Deng A, Zhang Y. Clinical characteristics and outcomes of patients with diabetes and COVID-19 in association with glucose-lowering medication. Diabetes Care. 2020.

34. Conversano A, Melillo F, Napolano A, Fominskiy E, Spessot M, Ciceri F, Agricola E. RAAs inhibitors and outcome in patients with SARS-CoV-2 pneumonia. A case series study. Hypertension. 2020.

35. de Abajo FJ, Rodríguez-Martín S, Lerma V, Mejía-Abril G, Aguilar M, García-Luque A, Laredo L, Laosa O, Centeno-Soto GA, Gálvez MÁ. Use of renin-angiotensin-aldosterone system inhibitors and risk of COVID-19 requiring admission to hospital: a case-population study. Lancet. 2020.

36. De Spiegeleer A, Bronselaer A, Teo JT, Byttebier G, De Tre G, Belmans L, Dobson R, Wynendaele E, Van De Wiele C, Vandaele F. The effects of ARBs, ACEIs and statins on clinical outcomes of COVID-19 infection among nursing home residents. medRxiv. 2020.

37. Ebinger JE, Achamallah N, Ji H, Claggett BL, Sun N, Botting P, Nguyen T-T, Luong E, Kim EH, Park E. Pre-existing 
characteristics associated with Covid-19 illness severity. medRxiv. 2020.

38. Felice C, Nardin C, Di Tanna GL, Grossi U, Bernardi E, Scaldaferri L, Romagnoli M, Tonon L, Cavasin P, Novello S. Use of RAAS inhibitors and risk of clinical deterioration in COVID-19: results from an Italian cohort of 133 hypertensives. Am J Hypertens. 2020;33:944-8.

39. Feng Z, Li J, Yao S, Yu Q, Zhou W, Mao X, Li H, Kang W, Ouyang $X$, Mei J. The Use of adjuvant therapy in preventing progression to severe pneumonia in patients with coronavirus disease 2019: a multicenter data analysis. medRxiv. 2020.

40. Gao C, Cai Y, Zhang K, Zhou L, Zhang Y, Zhang X, Li Q, Li W, Yang S, Zhao X. Association of hypertension and antihypertensive treatment with COVID-19 mortality: a retrospective observational study. Eur Heart J. 2020;41:2058-66.

41. Guo T, Fan Y, Chen M, Wu X, Zhang L, He T, Wang H, Wan J, Wang X, Lu Z. Cardiovascular implications of fatal outcomes of patients with coronavirus disease 2019 (COVID-19). JAMA Cardiol. 2020.

42. Hu J, Zhang X, Zhang X, Zhao H, Lian J, Hao S, Jia H, Yang M, Lu Y, Xiang D, Cai H, Zhang S, Gu J, Ye C, Yu G, Jin C, Zheng L, Yang Y, Sheng J. COVID-19 is more severe in patients with hypertension; ACEI/ARB treatment does not influence clinical severity and outcome. J Infect. 2020;81:979-97.

43. Huang Z, Cao J, Yao Y, Jin X, Luo Z, Xue Y, Zhu C, Song Y, Wang Y, Zou Y, Qian J, Yu K, Gong H, Ge J. The effect of RAS blockers on the clinical characteristics of COVID-19 patients with hypertension. Ann Transl Med. 2020 Apr;8(7):430. https://doi. org/10.21037/atm.2020.03.229. Erratum in: Ann Transl Med. 2020 Sep;8(17):1119. PMID: 32395474; PMCID: PMC7210199.

44. Huh K, Ji W, Kang M, Hong J, Bae GH, Lee R, Na Y, Choi H, Gong SY, Jung J. Association of previous medications with the risk of COVID-19: a nationwide claims-based study from South Korea. medRxiv. 2020.

45. Inciardi RM, Adamo M, Lupi L, Cani DS, Di Pasquale M, Tomasoni D, Italia L, Zaccone G, Tedino C, Fabbricatore D. Characteristics and outcomes of patients hospitalized for COVID-19 and cardiac disease in Northern Italy. Eur Heart J. 2020;41:1821-9.

46. Jung S-Y, Choi JC, You S-H, Kim W-Y. Association of reninangiotensin-aldosterone system inhibitors with COVID-19-related outcomes in Korea: a nationwide population-based cohort study. Clin Infect Dis. 2020.

47. Khawaja AP, Warwick AN, Hysi PG, Kastner A, Dick A, Khaw PT, Tufail A, Foster PJ, Khaw K-T. Associations with covid-19 hospitalisation amongst 406,793 adults: the UK Biobank prospective cohort study. medRxiv. 2020.

48. Khera R, Clark C, Lu Y, Guo Y, Ren S, Truax B, Spatz ES, Murugiah K, Lin Z, Omer SB, Vojta D, Krumholz HM. Association of angiotensin-converting enzyme inhibitors and angiotensin receptor blockers with the risk of hospitalization and death in hypertensive patients with coronavirus disease-19. medRxiv: the preprint server for health sciences 2020:2020.2005.2017.20104943.

49. Li J, Wang X, Chen J, Zhang H, Deng A. Association of reninangiotensin system inhibitors with severity or risk of death in patients with hypertension hospitalized for coronavirus disease 2019 (COVID-19) infection in Wuhan. JAMA cardiology: China; 2020.

50. Liu Y, Huang F, Xu J, Yang P, Qin Y, Cao M, Wang Z, Li X, Zhang S, Ye L. Anti-hypertensive Angiotensin II receptor blockers associated to mitigation of disease severity in elderly COVID19 patients. medRxiv. 2020.

51. Mancia G, Rea F, Ludergnani M, Apolone G, Corrao G. Reninangiotensin-aldosterone system blockers and the risk of Covid-19. N Engl J Med. 2020.

52. Meng J, Xiao G, Zhang J, He X, Ou M, Bi J, Yang R, Di W, Wang Z, Li Z. Renin-angiotensin system inhibitors improve the clinical outcomes of COVID-19 patients with hypertension. Emerg Microb Infect. 2020;9:757-60.

53. Peng Y, Meng K, Guan H, Leng L, Zhu R, Wang B, He M, Cheng L, Huang K, Zeng Q. Clinical characteristics and outcomes of 112 cardiovascular disease patients infected by 2019-nCoV. Zhonghua xin xue guan bing za zhi. 2020;48:E004-e004.

54. Rentsch CT, Kidwai-Khan F, Tate JP, Park LS, King JT, Skanderson M, Hauser RG, Schultze A, Jarvis CI, Holodniy M. Covid-19 testing, hospital admission, and intensive care among 2,026,227 United States veterans aged 54-75 years. MedRxiv. 2020.

55. Reynolds HR, Adhikari S, Pulgarin C, Troxel AB, Iturrate E, Johnson SB, Hausvater A, Newman JD, Berger JS, Bangalore $\mathrm{S}$. Renin-angiotensin-aldosterone system inhibitors and risk of Covid-19. N Engl J Med. 2020.

56. Richardson S, Hirsch JS, Narasimhan M, Crawford JM, McGinn $\mathrm{T}$, Davidson KW, Consortium at NC-R. presenting characteristics, comorbidities, and outcomes among 5700 patients hospitalized with COVID-19 in the New York City Area. JAMA. 2020;323:2052-2059.

57. Tedeschi S, Giannella M, Bartoletti M, Trapani F, Tadolini M, Borghi C, Viale P. Clinical impact of renin-angiotensin system inhibitors on in-hospital mortality of patients with hypertension hospitalized for COVID-19. Clin Infect Dis. 2020.

58. Yang G, Tan Z, Zhou L, Yang M, Peng L, Liu J, Cai J, Yang R, Han J, Huang Y. Angiotensin II receptor blockers and angiotensinconverting enzyme inhibitors usage is associated with improved inflammatory status and clinical outcomes in COVID-19 patients with hypertension. medRxiv. 2020.

59. Yu J SX, Ma J, et al. Long Term Use of ACEI/ARB contributes to the outcomes of COVID-19 patients with hypertension: a multicenter retrospective study. 2020.

60. Zeng Z, Sha T, Zhang Y, Wu F, Hu H, Li H, Han J, Song W, Huang Q, Chen Z. Hypertension in patients hospitalized with COVID-19 in Wuhan, China: a single-center retrospective observational study. medRxiv. 2020.

61. Zhang P, Zhu L, Cai J, Lei F, Qin J-J, Xie J, Liu Y-M, Zhao Y-C, Huang $X, \mathrm{Lin} \mathrm{L}$. Association of inpatient use of angiotensin converting enzyme inhibitors and angiotensin II receptor blockers with mortality among patients with hypertension hospitalized with COVID-19. Circul Res. 2020.

62. Zhou X, Zhu J, Xu T. Clinical characteristics of coronavirus disease 2019 (COVID-19) patients with hypertension on renin-angiotensin system inhibitors. Clin Exp Hypertens. 2019;2020:1-5.

63. Raisi-Estabragh Z, McCracken C, Ardissino M, Bethell MS, Cooper J, Cooper C, Harvey NC, Petersen SE. Non-white ethnicity, male sex, and higher body mass index, but not medications acting on the renin-angiotensin system are associated with coronavirus disease 2019 (COVID-19) hospitalisation: review of the first 669 cases from the UK biobank. medRxiv. 2020.

64. Choi HK, Koo H-J, Seok H, Jeon JH, Choi WS, Kim DJ, Park DW, Han E. ARB/ACEI use and severe COVID-19: a nationwide case-control study. medRxiv. 2020.

65. Chang TS, Ding Y, Freund MK, Johnson R, Schwarz T, Yabu JM, Hazlett C, Chiang JN, Wulf A, Geschwind DH. Prior diagnoses and medications as risk factors for COVID-19 in a Los Angeles Health System. medRxiv. 2020.

66. Schneeweiss MC, Leonard S, Weckstein A, Schneeweiss S, Rassen J. Renin-Angiotensin-Aldosterone-System inhibitor use in patients with COVID-19 infection and prevention of serious events: a cohort study in commercially insured patients in the US. medRxiv. 2020.

67. Kim JH, Baek Y-H, Lee H, Choe YJ, Shin HJ, Shin J-Y. Clinical Outcomes From COVID-19 Following Use of AngiotensinConverting Enzyme Inhibitors or Angiotensin-Receptor Blockers Among Patients with Hypertension in South Korea: a nationwide study. medRxiv. 2020. 
68. Saleknezhad N, Khosravi B, Anushiravani A, Eslahi M, Radmard AR, Sirusbakht A, Pourabbas SM, Abdollahi M, Kasaeian A, Sorouri M. COVID-19 and heart medications: what's the connection? medRxiv. 2020.

69. Georges J-L, Gilles F, Cochet H, Bertrand A, De Tournemire M, Monguillon V, Pasqualini M, Prevot A, Roger G, Saba J. Positive association of angiotensin II receptor blockers, not angiotensinconverting enzyme inhibitors, with an increased vulnerability to SARS-CoV-2 infection in patients hospitalized for suspected COVID-19 pneumonia. PLoS One. 2020;15:

70. Presta V, Figliuzzi I, Citoni B, Gallo G, Battistoni A, Tocci G, Volpe M. ARB-based combination therapy for the clinical management of hypertension and hypertension-related comorbidities: a spotlight on their use in COVID-19 patients. High Blood Press Cardiovasc Prev. 2021;28(3):255-62.

71. Triposkiadis F, Starling RC, Xanthopoulos A, Butler J, Boudoulas $\mathrm{H}$. Renin-angiotensin-system inhibition in the context of corona virus disease-19: experimental evidence, observational studies, and clinical implications. Heart Fail Rev. 2021 Mar;26(2):381-389. https://doi.org/10.1007/s10741-020-10022-4. Epub 2020 Sep 1. PMID: 32875490; PMCID: PMC7462660.
72. Iaccarino G, Grassi G, Borghi C, Ferri C, Salvetti M, Volpe M; SARS-RAS Investigators. Age and Multimorbidity Predict Death Among COVID-19 Patients: Results of the SARS-RAS study of the Italian society of hypertension. Hypertension. 2020;76(2):366-372.

73. Twerenbold R. The COVIVA Study. https://clinicaltrials.gov/ct2/ show/NCT04366765.

74. Mach F. The Geneva Covid-19 CVD Study. https://clinicaltrials. gov/ct2/show/NCT04384029.

75. Gracia-Ramos AE. Telmisartan in Respiratory Failure Due to COVID-19. https://clinicaltrials.gov/ct2/show/NCT04510662.

76. Miklos Lipcsey P. COVID-19 in the Swedish ICU-cohort: risk factors of critical care admission and intensive care mortality. https://www.clinicaltrials.gov/ct2/show/NCT04390074.

77. Jean-Louis GEORGES M, MPH. Renin-Angiotensin-Aldosterone System Inhibitors, Hypertension, and COVID-19 (COVHYP). https://clinicaltrials.gov/ct2/show/NCT04374695.

78. Abhinav Sharma M. The McGill RAAS-COVID-19 Trial (RAASCOVID). https://clinicaltrials.gov/ct2/show/NCT04508985. 